\title{
Fingerprint Classification using Fast Fourier Transform and Nonlinear Discriminant Analysis*
}

\author{
Cheong Hee Park ${ }^{\dagger}$ and Haesun Park ${ }^{\ddagger}$ \\ Dept. of Computer Science and Engineering \\ University of Minnesota \\ Minneapolis, MN 55455, USA \\ \{chpark, hpark\}@cs.umn.edu
}

\begin{abstract}
In this paper, we present a new approach for fingerprint classification based on Discrete Fourier Transform (DFT) and nonlinear discriminant analysis. Utilizing the Discrete Fourier Transform and directional filters, a reliable and efficient directional image is constructed from each fingerprint image, and then nonlinear discriminant analysis is applied to the constructed directional images, reducing the dimension dramatically and extracting the discriminant features. The proposed method explores the capability of DFT and directional filtering in dealing with low quality images and the effectiveness of nonlinear feature extraction method in fingerprint classification. Experimental results demonstrates competitive performance compared with other published results.
\end{abstract}

Keywords Discrete Fourier Transform, Fingerprint classification, Generalized singular value decomposition, Nonlinear discriminant analysis, Kernel methods.

${ }^{*}$ This work was supported in part by the National Science Foundation grants CCR-0204109 and ACI0305543. Any opinions, findings and conclusions or recommendations expressed in this material are those of the authors and do not necessarily reflect the views of the National Science Foundation(NSF).

${ }^{\dagger}$ Corresponding author: Tel: 1-352-392-9011, Fax: 1-352-392-3537. C. H. Park is currently as a postdoc at the Dept. of Industrial and Systems Engineering, University of Florida, Gainesville, FL 32611, USA.

${ }^{\ddagger}$ H. Park is in the National Science Foundation, 4201 Wilson Boulevard, Arlington, Virginia 22230, USA. The work of H. Park has been performed while at the NSF and was supported by IR/D from the NSF. 


\section{Introduction}

Fingerprints have long been used as a reliable biometric feature for personal identification. Fingerprint classification refers to the problem of assigning fingerprints to one of several prespecified classes. Automatic classification can be used as a preprocessing step for fingerprint matching, reducing matching time and complexity by narrowing the search space to a subset of a typically huge database [1,2]. Fingerprints are graphical images of flow-like ridges, and they are classified as left loop (L), right loop (R), whorl (W), arch (A) and tented arch (T) according to Henry's classification scheme [3] as shown in Fig. 1.

Classification performance highly depends on the preprocessing steps where various ways to extract and represent distinguishable features among classes can be applied. The features generated after the preprocessing steps are fed into classifiers such as neural networks [4], hidden Markov model [5], probabilistic neural networks [6], support vector machines [7]. Jain et al. extracted features from Gabor-filtered images which were centered on a core point and used two-stage classifiers of K-nearest neighbor and neural networks [2]. Since the fingerprints are flow-like images, many approaches are based on directional structures explicitly or implicitly. Local orientational flows are estimated from a fingerprint image and desired features are computed from the estimated orientation field. The features can be singular points and the classification is based on the number and locations of detected singular points [8]. In [9], a directional image is partitioned into homogeneous regions and the relative relations of segmented regions are used for classification. In order to overcome the possible ill-posed problem of the directional partitioning task, Cappelli et al. performed guided segmentation using directional masks as a prototype of each class [10].

In this paper, we propose an approach for fingerprint classification based on the Discrete Fourier Transform (DFT) and nonlinear discriminant analysis. In order to obtain reliable and efficient representations of fingerprint images, we utilize the Discrete Fourier 
Transform (DFT) and directional filters. The DFT is a useful computational tool that provides an efficient means for detecting directionality or periodicity in the frequency domain and removing noise by deleting high frequency coefficients [11]. In order to estimate local ridge orientations in a fingerprint image, we apply directional filters in the frequency domain after the image has been transformed by the DFT. Directional images are then constructed in order to capture the distinguishing orientational structure among classes. By deleting high frequency coefficients in the frequency domain, our method utilizing the DFT and directional filters can deal with noise in fingerprint images effectively, and therefore tolerate low quality fingerprint images. Kernel discriminant analysis (KDA/GSVD) is a nonlinear discriminant analysis based on kernel methods and the generalized singular value decomposition (GSVD) [12]. It performs Linear Discriminant Analysis after transforming the original data to a linearly separable structure using a kernel-based nonlinear mapping. KDA/GSVD is capable of handling high dimensional data and extracting most discriminant features for classification automatically. We apply KDA/GSVD to the constructed directional images extracting nonlinear discriminant features, which are used for very effective fingerprint classification.

The paper is organized as follows. In Section 2, we give a detailed explanation of how to construct directional images using the DFT and directional filters. A nonlinear discriminant analysis, KDA/GSVD, based on kernel methods and the GSVD is presented in Section 3. Experimental results using the NIST database 4 [13] demonstrates the comparable performance of our proposed method over previously proposed methods.

\section{Construction of Directional Images}

Fingerprints vary in size, are positioned randomly in printed images, and the backgrounds can contain noise such as scratches, lines or blur. In the Discrete Fourier Transform (DFT), 
thresholding the high frequency coefficients corresponds to reducing the noise effects, while the low frequency coefficients provide a trigonometric interpolation via a finite linear combination of sines and cosines of the various frequencies [14]. The DFT has been widely used in signal and image processing [11]. We apply the Fast Fourier Transform (FFT), the fast algorithm for computing the DFT, to construct a directional image.

Let $I(x, y)$ denote the gray level at $(x, y)$ in an $N \times N$ image. The image $I$ in the spatial domain is transformed to the frequency domain by the 2-dimensional FFT,

$$
F(k, l)=\sum_{m=0}^{N-1} \sum_{n=0}^{N-1} I(m, n) \exp \left\{-\sqrt{-1} \frac{2 \pi}{N} k m\right\} \exp \left\{-\sqrt{-1} \frac{2 \pi}{N} \ln \right\},
$$

for $0 \leq k, l \leq N-1$. By translating the image $F$ by $\left(\frac{N}{2}, \frac{N}{2}\right)$ and wrapping it around at the edges, the zero frequency (DC point - DC stands for Direct Current) is moved to the point $\left(\frac{N}{2}, \frac{N}{2}\right)$ in the frequency domain and the absolute magnitude image in the frequency domain becomes symmetric around the DC point. In the rest of the paper, we will assume that FFT has been applied and we proceed in the shifted frequency domain. Absolute magnitudes along the line passing through the DC point in the frequency domain can be viewed as responses of sine and cosine waves of the same orientation but at various frequencies in the space domain. FFT transformed images in the frequency domain are shown in Fig. 2. The first FFT image in Fig. 2 shows a high response along the line of about $135^{\circ}$ passing through the DC point, indicating the strong ridge orientation of $45^{\circ}$ in the corresponding square image of the fingerprint. By using directional filters, we can find the dominant direction and its directionality.

The main steps for constructing a directional image are:

(1) Segment the fingerprint from the background by applying the FFT in a local neighborhood and computing the directionality.

(2) On a segmented fingerprint, compute the directional vectors by computing the dominant directions in a local neighborhood. 
(3) Find the core point, to be defined later, which can be used as a landmark for unified centering of fingerprints belonging to the same class, and construct the directional image centered on the core point.

As we explain the three steps in the next sections, the specific parameter values used in our experiments are given. The parameter values were determined by applying the three steps to fingerprint images of the training data set and examining the produced directional images.

\subsection{Fingerprint Segmentation}

Given an input image of size $512 \times 512$, its rows and columns are divided into 16 pixels, giving $31 \times 31$ inner grid points. The FFT is applied on the $32 \times 32$ pixels centered at each inner grid point $(m, n), 1 \leq m, n \leq 31$. See the square regions in the fingerprint image and the corresponding FFT images in Fig. 2. In order to detect the dominant direction of orientational flow in each $32 \times 32$ sized local neighborhood, we devise a filter, called a directional filter, which will be used in the FFT image. The directional filter $D_{\theta}$ for orientation $\theta \in[0,180)$ is given as follows:

$$
\begin{aligned}
D_{\theta}(i+p, j+p)= & \left\{\begin{array}{c}
\exp \left\{-\frac{u^{2}}{a^{2}}-\frac{v^{2}}{b^{2}}\right\}, \quad \text { if } \frac{u^{2}}{a^{2}}+\frac{v^{2}}{b^{2}} \leq 1, \\
0, \quad \text { otherwise, }
\end{array} \text { for }-p \leq i, j \leq p,\right. \\
\text { where } & \left\{\begin{array}{l}
v=i * \cos \theta+j * \sin \theta \\
u=\sqrt{i^{2}+j^{2}-v^{2}},
\end{array}\right.
\end{aligned}
$$

$2 \times a$ and $2 \times b$ are the lengths of the short and the long axes of the ellipsoid $\frac{u^{2}}{a^{2}}+\frac{v^{2}}{b^{2}}=1$, respectively. An example of directional filters is illustrated in Fig. 3. By using directional filters that emphasize the low frequency coefficients around the DC point and disregard the high frequency coefficients, the noise effects in fingerprint images can be reduced effectively. In our algorithm, we used $p=16, a=1.5, b=32 / 3$ and $\theta=0,10, \cdots, 170$.

At each grid point $(m, n), 1 \leq m, n \leq 31$, directionality $\mathcal{D}_{m, n}$ and local dominant 
direction $\theta_{m, n} \equiv \theta_{\max }$ are calculated as

$$
\begin{aligned}
& \mathcal{D}_{m, n}=\underbrace{}_{\theta \quad \frac{f\left(\theta_{\text {max }}\right)-f(\theta)}{f\left(\theta_{\text {max }}\right)}} \\
& \text { where } \quad f(\theta)={ }_{i=0}^{2 p \quad 2 p} D_{\theta=0}(i, j) \times|F(i, j)|, \quad \theta_{\text {max }}=\operatorname{argmax}_{\theta} f(\theta) .
\end{aligned}
$$

The value $f(\theta)$ is the weighted sum of FFT coefficients by the directional filter $D_{\theta}$ along the direction $\theta$, and $\mathcal{D}_{m, n}$ measures how strong the direction $\theta_{\text {max }}$ stands out. After computing $\left\{\left(\mathcal{D}_{m, n}, \theta_{m, n}\right) \mid 1 \leq m \leq 31,1 \leq n \leq 31\right\}$, by thresholding out the elements with low directionality $\mathcal{D}_{m, n}$ or horizontal or vertical direction $\theta_{m, n}$ and then choosing the outermost rows and columns that have the remaining elements with magnitude greater than 1 , the boundaries for segmentation of foreground fingerprint from the background plane are determined. We used the mean value of $\mathcal{D}_{m, n}, 1 \leq m, n \leq 31$ as a threshold.

\subsection{Computation of Directional Vectors}

The size of the segmented fingerprints can vary. In order to obtain scaling invariance, we divide the segmented fingerprint to give $31 \times 31$ inner grids and the FFT is applied to $32 \times 32$ local neighborhoods centered at each grid point. The local dominant directions are detected as $\theta_{\max }$ given in (4). The obtained dominant direction $\theta$ is represented as a vector $[\cos (2 \theta), \sin (2 \theta)]$ where $0 \leq \theta \leq 180$ to avoid the discontinuity between 0 and 180 as in [6]. Let

$$
d_{m, n}=\left[\cos \left(2 \theta_{m, n}\right), \sin \left(2 \theta_{m, n}\right)\right]
$$

be the directional vectors at $1 \leq m, n \leq 31$. Smoothing of the directional elements is done by averaging over a $3 \times 3$ neighborhood as follows.

$$
\begin{aligned}
& \tilde{d}_{m, n}=\left[\cos \left(2 \tilde{\theta}_{m, n}\right), \sin \left(2 \tilde{\theta}_{m, n}\right)\right], \\
& \text { where } \quad \tilde{\theta}_{m, n}=\frac{1}{2} \arctan \left(\frac{y}{x}\right) \quad \text { and }[x, y]={ }_{k=-1 l=-1}^{1} d_{m+k, n+l} .
\end{aligned}
$$




\subsection{Construction of Directional Image}

Due to the variance in size and positions of the printed fingerprints, the computed directional array $\left\{\tilde{d}_{m, n} \mid 1 \leq m \leq 31,1 \leq n \leq 31\right\}$ needs to be adjusted to increase consistency for fingerprints belonging to the same class. It can be done by detecting a point which is common in fingerprints within each class, but unique to characterize each class, and then by extracting an area centered at the point, which is called the core point.

The core point is detected by measuring the consistency of directional elements over $3 \times 3$ neighborhood by

$$
u_{m, n}=\left|\begin{array}{l}
1 \\
k=-1 l=-1 \\
\tilde{d}_{m+k, n+l}
\end{array}\right|
$$

at $(m, n)$. It measures the distance from the starting point to the finishing point after adding directional vectors within a neighborhood. The lower value indicates inconsistency of directions over a neighborhood, as in the central point of swirling circles in the fingerprints of the class whorl (W) or a rapid turning point in orientational flows of ridges and valleys. In order to find the core point, the lowest value among

$$
{ }_{k=-1 l=-1}^{0} u_{m+k, n+l}
$$

is searched in $11 \leq m, n \leq 21$. This core point is used to locate the area which is most common within classes and discriminant between classes. Most of the information about the directional structure of a fingerprint can be found around the core point. In our experiments, $21 \times 21$ directional vectors centered on the core point were used. From the array of size $21 \times 21$ of directional vectors, a directional image is constructed where each directional vector $\tilde{d}_{m, n}$ in (5) is drawn based on $M_{\tilde{\theta}_{m, n}}$ of size $5 \times 5$ as shown in Fig. 4, resulting in the 
directional map of size $105 \times 105$. The formula for $M_{\theta}$ is given as

$$
\begin{aligned}
& M_{\theta}(i+p, j+p)=\begin{array}{c}
C \cdot \exp \left\{-\frac{u^{2}}{a^{2}}\right\}, \\
0,
\end{array} \begin{array}{c}
\text { if } \frac{u^{2}}{a^{2}} \leq 1, \\
\text { otherwise, }
\end{array} \quad \text { for }-p \leq i, j \leq p, \\
& \text { where } \quad \begin{array}{l}
v=i * \cos \theta+j * \sin \theta \\
u=\frac{i^{2}+j^{2}-v^{2}}{i^{2}}
\end{array}
\end{aligned}
$$

Here we used $p=2, a=1.2$ and $C=10$. In Fig. 5, the constructed directional images corresponding to the fingerprints in Fig. 1 are shown, where the detected core points are located in the center.

In the next section, we present nonlinear discriminant analysis based on kernel methods and the generalized singular value decomposition (GSVD), called KDA/GSVD [12]. As Linear Discriminant Analysis (LDA) finds a linear transformation which maximizes distances between classes and minimizes scatter within classes, KDA/GSVD performs LDA on the feature space transformed by a kernel-based nonlinear mapping. A nonlinear mapping via a kernel function makes LDA successfully find a linear transformation to maximally separate classes, even if the original data is not linearly separable.

\section{Kernel Discriminant Analysis based on Generalized Sin- gular Value Decomposition}

We assume the vector space representation of a data set $A$

$$
A=\left[a_{1}, \cdots, a_{n}\right]=\left[A_{1}, \cdots, A_{r}\right] \in \mathbb{R}^{m \times n}
$$

where each data item in an $m$-dimensional space is represented as a column vector $a_{i}$ and $A$ is grouped into $r$ classes, $A_{i}, 1 \leq i \leq r$. For our fingerprint classification, the constructed directional images shown in Fig. 5 correspond to data items. Since the size of a directional image is $105 \times 105=11025$, the data diemension $m$ is 11025 . Each class $A_{i}$ has $n_{i}$ elements and $N_{i}$ denotes the set of column indices in each class $A_{i}$. Then the between-class scatter 
matrix $S_{b}$ and the within-class scatter matrix $S_{w}$ are defined as

$$
\begin{aligned}
S_{b} & ={ }_{i=1}^{r} n_{i}\left(c_{i}-c\right)\left(c_{i}-c\right)^{T} \in \mathbb{R}^{m \times m} \text { and } \\
S_{w} & ={ }_{i=1}^{r}\left(a_{j}-c_{i}\right)\left(a_{j}-c_{i}\right)^{T} \in \mathbb{R}^{m \times m},
\end{aligned}
$$

where $c_{i}$ is the centroid of the $i$-th class and $c$ is the global centroid.

Linear Discriminant Analysis (LDA) searches for a linear transformation which maximizes the between-class scatter and minimizes the within-class scatter. It is known [15] that the optimal solution for LDA is obtained by the eigenvectors $x$ corresponding to the $r-1$ largest eigenvalues $\lambda$ of

$$
S_{w}^{-1} S_{b} x=\lambda x
$$

However, when the data dimension $m$ is larger than the number of data $n$, the withinclass scatter matrix $S_{w}$ becomes singular and $S_{w}^{-1}$ is not defined. Recently, a method called LDA/GSVD has been developed which is a generalization of LDA based on the generalized singular value decomposition (GSVD) $[16,17]$. By using the generalized singular value decomposition (GSVD), LDA/GSVD solves a generalized eigenvalue problem

$$
S_{b} x=\lambda S_{w} x
$$

regardless of the singularity of scatter matrices. Note that $S_{b}$ and $S_{w}$ can be expressed as

$$
\begin{aligned}
& S_{b}=H_{b} H_{b}^{T}, \quad S_{w}=H_{w} H_{w}^{T} \text { where } \\
& H_{b}=\left[\sqrt{n_{1}}\left(c_{1}-c\right), \cdots, \sqrt{n_{r}}\left(c_{r}-c\right)\right] \in \mathbb{R}^{m \times r}, \\
& H_{w}=\left[A_{1}-c_{1} e_{1}, \cdots, A_{r}-c_{r} e_{r}\right] \in \mathbb{R}^{m \times n}, \\
& e_{i}=[1, \cdots, 1] \in \mathbb{R}^{1 \times n_{i}} .
\end{aligned}
$$

The GSVD is applied to the pair $\left(H_{b}^{T}, H_{w}^{T}\right)$, diagonalizing $H_{b}^{T}$ and $H_{w}^{T}$ as

$$
U^{T} H_{b}^{T} X=\left[\Sigma_{b}, 0\right] \quad \text { and } \quad V^{T} H_{w}^{T} X=\left[\Sigma_{w}, 0\right]
$$


where $U$ and $V$ are orthogonal, $X$ is nonsingular, and $\Sigma_{b}$ and $\Sigma_{w}$ are diagonal matrices. The matrix $\Sigma_{b}$ has nonincreasing diagonal elements and $\Sigma_{w}$ has nondecreasing diagonal elements. From (10) and (11), we have

$$
X^{T} S_{b} X=\left[\begin{array}{cc}
\Sigma_{b}^{T} \Sigma_{b} & 0 \\
0 & 0
\end{array}\right] \quad \text { and } \quad X^{T} S_{w} X=\left[\begin{array}{cc}
\Sigma_{w}^{T} \Sigma_{w} & 0 \\
0 & 0
\end{array}\right]
$$

obtaining the leftmost $r-1$ columns of $X$ for the LDA solution. The algorithm to compute $X$ by the GSVD can be found in [16].

Although the LDA can find the optimal transformation to preserve the cluster structure, it can not overcome the limitation due to non-linearly separable data. In order to make LDA applicable to nonlinearly separable data, a kernel-based nonlinear extension of LDA has been proposed $[18,19,20,21,12]$. The main idea of kernel-based methods is to map the input data to a feature space by a nonlinear mapping through a kernel function, where inner products in the feature space can be computed by a kernel function without knowing the nonlinear mapping explicitly. When the input space is transformed by a kernel-based nonlinear mapping, the dimension of the feature space becomes much larger (possibly infinite) than that of the input space, and as a result, the scatter matrices become singular in the feature space. For our fingerprint classification, we use a nonlinear Discriminat Analysis algorithm, called KDA/GSVD, which conducts LDA using the GSVD in the transformed feature space.

Given a kernel function $\kappa(x, y)$ satisfying Mercer's condition [22], there exists a nonlinear mapping $\Phi$ from the original data space $A$ to a feature space $\mathcal{F}$ such that inner products of $\Phi(x)$ and $\Phi(y)$ in the feature space $\mathcal{F}$ can be computed by

$$
<\Phi(x), \Phi(y)>=\kappa(x, y)
$$

Let $\mathcal{S}_{b}$ and $\mathcal{S}_{w}$ be the between-class and within-class scatter matrices in $\mathcal{F}$. Then the optimal 


\section{Algorithm 1 KDA/GSVD}

Given a data matrix $A=\left[a_{1}, \cdots, a_{n}\right] \in \mathbb{R}^{m \times n}$ with $r$ classes and a kernel function $\kappa$, it computes the $r-1$ dimensional representation $y$ of any input vector $x \in \mathbb{R}^{m \times 1}$.

1. Compute $\mathcal{K}_{b} \in \mathbb{R}^{n \times r}$ and $\mathcal{K}_{w} \in \mathbb{R}^{n \times n}$ according to Eqns. in (16).

2. Apply the GSVD to the pair $\left(\mathcal{K}_{b}^{T}, \mathcal{K}_{w}^{T}\right)$ to obtain

$$
\mathcal{U}^{T} \mathcal{K}_{b}^{T} \mathcal{X}=\left[\Gamma_{b}, 0\right] \quad \text { and } \quad \mathcal{V}^{T} \mathcal{K}_{w}^{T} \mathcal{X}=\left[\Gamma_{w}, 0\right] .
$$

3. Assign the first $r-1$ columns of $\mathcal{X}$ to $\mathcal{G}: \mathcal{G}=\left[\tilde{\alpha}^{(1)}, \cdots, \tilde{\alpha}^{(r-1)}\right] \in \mathbb{R}^{n \times(r-1)}$.

4. For any input vector $x \in \mathbb{R}^{m \times 1}$, a dimension reduced representation $y$ is computed as

$$
y=\mathcal{G}^{T}\left[\begin{array}{c}
\kappa\left(a_{1}, x\right) \\
\vdots \\
\kappa\left(a_{n}, x\right)
\end{array}\right] .
$$

transformation for LDA can be found by solving

$$
\mathcal{S}_{b} \psi=\lambda \mathcal{S}_{w} \psi
$$

As in (10), $\mathcal{S}_{b}$ and $\mathcal{S}_{w}$ can be expressed with $\mathcal{H}_{b}$ and $\mathcal{H}_{w}$,

$$
\begin{aligned}
& \mathcal{S}_{b}=\mathcal{H}_{b} \mathcal{H}_{b}^{T}, \quad \mathcal{S}_{w}=\mathcal{H}_{w} \mathcal{H}_{w}^{T}, \quad \text { where } \\
& \mathcal{H}_{b}=\left[\sqrt{n_{1}}\left(c_{1}^{\Phi}-c^{\Phi}\right), \cdots, \sqrt{n_{r}}\left(c_{r}^{\Phi}-c^{\Phi}\right)\right], \\
& \mathcal{H}_{w}=\left[\Phi\left(A_{1}\right)-c_{1}^{\Phi} e_{1}, \cdots, \Phi\left(A_{r}\right)-c_{r}^{\Phi} e_{r}\right],
\end{aligned}
$$

and $c^{\Phi}$ and $c_{i}^{\Phi}$ are the global centroid and the $i$-th class centroid in the feature space $\mathcal{F}$, respectively. Note that Eqns. in (15) can not be computed explictly, since the nonlinear mapping $\Phi$ is not known explicitly. However, as long as the problem is formulated in terms of inner products, we can work on the feature space $\mathcal{F}$ by (13). 
Representing $\psi=\sum_{i=1}^{n} \alpha_{i} \Phi\left(a_{i}\right)$, we can derive the following equations

$$
\begin{aligned}
& \mathcal{H}_{b}^{T} \psi=\mathcal{K}_{b}^{T} \tilde{\alpha}, \quad \mathcal{H}_{w}^{T} \psi=\mathcal{K}_{w}^{T} \tilde{\alpha} \quad \text { where } \tilde{\alpha}=\left[\alpha_{1}, \cdots, \alpha_{n}\right]^{T}, \\
& \mathcal{K}_{b}=\left[\frac{\sqrt{n_{j}}}{n_{j}} \sum_{s \in N_{j}} \kappa\left(a_{s}, a_{i}\right)-\frac{\sqrt{n_{j}}}{n} \sum_{s=1}^{n} \kappa\left(a_{s}, a_{i}\right)\right]_{(1 \leq i \leq n, 1 \leq j \leq r)}, \\
& \mathcal{K}_{w}=\left[\kappa\left(a_{i}, a_{j}\right)-\frac{1}{n_{s}} \sum_{t \in N_{s}} \kappa\left(a_{t}, a_{i}\right)\right]_{(1 \leq i \leq n, 1 \leq j \leq n)} .
\end{aligned}
$$

The problem is now to solve the generalized eigenvalue problem

$$
\mathcal{K}_{b} \mathcal{K}_{b}^{T} \tilde{\alpha}=\lambda \mathcal{K}_{w} \mathcal{K}_{w}^{T} \tilde{\alpha}
$$

By applying the GSVD to the pair $\left(\mathcal{K}_{b}^{T}, \mathcal{K}_{w}^{T}\right)$, we can find the desired $r-1$ eigenvectors $\tilde{\alpha}^{(1)}, \cdots, \tilde{\alpha}^{(r-1)}$ of (17) which give the solutions $\psi_{1}, \cdots, \psi_{r-1}$ of (14). The algorithm KDA/GSVD is summarized in Algorithm 1. See [12] for a detailed discussion for the KDA/GSVD method.

\section{Experimental Results}

The NIST database 4 is a collection of 4000 fingerprint images of size $512 \times 512$. They have been classified into 5 classes, left loop (L), right loop (R), whorl (W), arch (A) and tented arch (T) by human experts. For $17.5 \%$ of 4000 fingerprints, two class labels have been assigned due to a variety of ambiguities such as a scar occurring in the fingerprints, the quality of the print rolling, and the print having a ridge structure characteristic of two different classes [13]. Table 1 shows the distribution of 4000 fingerprints. Each row sums up to 800 according to the classification based on the first class label, while the natural distribution is estimated to be $0.317,0.338,0.279,0.037$ and 0.029 for $\mathrm{L}, \mathrm{R}, \mathrm{W}, \mathrm{A}$ and $\mathrm{T}$, respectively. Since the prediction for class $\mathrm{T}$ is considered most difficult and its natural distribution is relatively small compared with classes $\mathrm{L}, \mathrm{R}$ and $\mathrm{W}$, the uniform distribution in the NIST database 4 makes the classification more challenging. Within each row in Table 1, the distribution of fingerprints which are cross-referenced to the second class 
is shown. In handling the cross-referenced fingerprints, one can consider only the first class label disregrading the second class label. However, considering that the classification was difficult even by human experts, in our experiment a fingerprint was considered as belonging to both classes when it has multiple class labels.

Among the 4000 fingerprint images, 2000 fingerprints, S0001 to S2000, are the second rolling of the same people from whom fingerprints F0001 to F2000 were taken. For a fair comparison, we set experimental conditions the same as those in $[2,7]$. The training set is formed with the first 1000 fingerprints from $\mathrm{F}$ labels and the first 1000 fingerprints from S labels, and the test set is composed of the remaining 2000 fingerprints. If the predicted class label for the test data matches one of the multiple labels, the prediction is considered to be correct.

Following the procedures of Section 2, we constructed directional images of 4000 fingerprints. In this experiment, we used a Gaussian Kernel for KDA/GSVD

$$
\kappa(x, y)=\exp \left(-\frac{\|x-y\|^{2}}{2 \sigma^{2}}\right), \sigma \in \mathbb{R}
$$

where the value for $\sigma$ was set to be 0.5 multiplied by the average of pairwise distances in the training data. Since there are five classes, the dimension is dramatically reduced from $105 \times 105$ to 4 by KDA/GSVD. We applied centroid-based classification in the reduced dimensional space, where each test datum is assigned to the class of which the centroid is the nearest to the test datum. Table 2 shows the prediction accuracies of our method as well as the results from the two-stage classifiers of [2] and support vector machines (SVM) [7]. The prediction accuracies by other classifiers such as multilayer perceptron (MLP), recursive neural networks $(\mathrm{RNN})$ and $k$-nearest neighbors $(k-\mathrm{NN})$ classifiers can be found in [7] where the best prediction accuracy among them was achieved by SVM. Since Jain et al. made a rejection at the step of extracting feature vectors when the center point was detected at a corner of the image, the prediction accuracy with 0 percent rejection 
rate for their method is not available. In our method, the rejection criterion is based on the ratio between the distance to the nearest class centroid and the distance to the farthest class centroid. The confusion matrix by our proposed method is shown in Table 3 . In the confusion matrix, when the test data is cross-referenced and misclassified, the first class label was considered the actual class label. The proposed method obtained high prediction accuracies even with a simple centroid-based classifier. Since the dimension is reduced so dramatically, in the test stage the savings of computational complexity can be achieved.

In order to verify that a nonlinear discriminant analysis works efficiently in fingerprint classification, we utilized a NIST fingerprint image software PCASYS [6]. The PCASYS extracts a two dimensional array of size $28 \times 30$ of orientation vectors $[\cos (2 \theta), \sin (2 \theta)]$ from a fingerprint image after various preprocessing. (See [6] for details.) The array of the size $28 \times 30$ by PCASYS corresponds to an array of the $21 \times 21$ directional vectors of Section 2.3 in our proposed method. From the arrays extracted by PCASYS, directional images were constructed by using $M_{\theta}$ in (7), and then KDA/GSVD was applied to the constructed directional images. The prediction accuracies are shown on the second line (PCASYS + KDA/GSVD) in Table 4 which repeats the results from Table 2 for comparison. This demonstrates that the nonlinear feature extraction by kernel discriminant analysis can be used effectively to extract optimal features for fingerprint classification.

We also applied KDA/GSVD for the four-class classification problem where the classes $\mathrm{A}$ and $\mathrm{T}$ are combined. Since the number of classes in this problem is 4 , the reduced dimension by KDA/GSVD is 3 . The results by our method and from $[2,7]$ are reported in Table 5. 


\section{Conclusion}

In this paper, a novel approach was presented for fingerprint classification based on Discrete Fourier Transform and nonlinear discriminant analysis. The directional images are constructed from fingerprint images utilizing the Discrete Fourier Transform (DFT). Applying directional filters in the frequency domain after the transformation by the DFT achieves effective low frequency filtering, reducing the noise effects in fingerprint images. The constructed directional images contain the essential directional structure which is common within each class and discriminates between classes. Kernel-based nonlinear discriminant analysis performs dramatic dimension reduction giving high quality discriminant information for classification by capturing global difference among classes. The fast algorithm FFT for DFT speeds up the preprocessing to construct directional images. Once the transformation matrix by KDA/GSVD is computed, the classification in the reduced dimensional space saves computational complexites further. The experimental results show that even simple classification methods such as centroid-based classification can achieve high prediction accuracies over more complex methods previously published.

\section{Acknowledgements}

The authors would like to thank Dr. Panos Pardalos for valuable comments and informations.

\section{References}

[1] H.C. Lee and R.E. Gaensslen. Advances in Fingerprint Technology. Elsevier, 1991.

[2] A.K. Jain, A. Prabhakar, and L. Hong. A multichannel approach to fingerprint classification. IEEE transactions on pattern analysis and machine intelligence, 
21(4):348-359, 1999.

[3] E.R. Henry. Classification and Uses of Finger Prints. London: Routledge, 1900.

[4] K. Moscinska and G. Tyma. Neural network based fingerprint pattern classification. Proc. Third Int'l Conf. Neural Network, pages 229-232, 1993.

[5] A. Senior. A hidden markov model fingerprint classifier. Proc. 31st Asilomar Conf. Signals, Systems, and Computers, pages 306-310, 1997.

[6] G.T. Candela, P.J. Grother, C.I. Watson, R.A. Wilkinson, and C.L. Wilson. PCASYSa pattern-level classification automation system for fingerprints, 1995. NISTIR 5647, Aug.

[7] Y. Yao, G.L. Marcialis, M. Pontil, P. Frasconi, and F. Roli. Combining flat and structured representations for fingerprint classification with recursive neural networks and support vector machines. Pattern Recognition, 36(2):397-406, 2003.

[8] K. Karu and A.K. Jain. Fingerprint classification. Pattern Recognition, 29(3):389404, 1996.

[9] D. Maio and D. Maltoni. A structural approach to fingerprint classification, 1996. Proc. 13th ICPR, Vienna, Aug.

[10] R. Cappelli, A. Lumini, and D. Maio. Fingerprint classification by directional image partitioning. IEEE transactions on pattern analysis and machine intelligence, 21(5):402-421, 1999.

[11] F. Liu and R.W. Picard. Periodicity, directionality, and randomness: Wold features for image modeling and retrieval. IEEE transactions on pattern analysis and machine intelligence, 18(7):722-733, 1996. 
[12] C.H. Park and H. Park. Kernel discriminant analysis based on generalized singular value decomposition. Technical Reports 03-017, Department of Computer Science and Engineering, University of Minnesota, Twin Cities, 2003.

[13] C.I. Watson and C.L. Wilson. NIST special database 4. fingerprint database. National Institute of Standard and Technology(March 1992).

[14] A.V. Oppenheim, A.S. Willsky, and I.T. Young. Signals and Systems. Prentice-Hall, 1983.

[15] K. Fukunaga. Introduction to Statistical Pattern Recognition. Acadamic Press, second edition, 1990.

[16] P. Howland, M. Jeon, and H. Park. Structure preserving dimension reduction for clustered text data based on the generalized singular value decomposition. SIAM Journal on Matrix Analysis and Applications, 25(1):165-179, 2003.

[17] P. Howland and H. Park. Generalizing discriminant analysis using the generalized singular value decomposition. IEEE transactions on pattern analysis and machine intelligence, to appear. Also available in Technical Reports 03-013, Department of Computer Science and Engineering, University of Minnesota, Twin Cities, 2003.

[18] S. Mika, G. Rätsch, J. Weston, B. Schölkopf, and K.-R. Müller. Fisher discriminant analysis with kernels. In E.Wilson J.Larsen and S.Douglas, editors, Neural networks for signal processing IX, pages 41-48. IEEE, 1999.

[19] G. Baudat and F. Anouar. Generalized discriminant analysis using a kernel approach. Neural computation, 12:2385-2404, 2000.

[20] V. Roth and V. Steinhage. Nonlinear discriminant analysis using kernel functions. Advances in neural information processing systems, 12:568-574, 2000. 
[21] S.A. Billings and K.L. Lee. Nonlinear fisher discriminant analysis using a minimum squared error cost function and the orthogonal least squares algorithm. Neural networks, 15(2):263-270, 2002.

[22] N. Cristianini and J. Shawe-Taylor. An Introduction to Support Vector Machines and other kernel-based learning methods. Cambridge, 2000.

About the Author- Cheong Hee Park received her Ph.D. in Mathematics from Yonsei University, Korea in 1998. She received the M.S. and Ph.D. degrees in Computer Science at the Department of Computer Science and Engineering, University of Minnesota in 2002 and 2004 respectively. Her research interests include pattern recognition, data mining, bioinformatics and machine learning.

About the Author-Haesun Park received her B.S. degree in Mathematics from Seoul National University, Seoul Korea, in 1981 with summa cum laude and the university president's medal as the top graduate of the university. She received her M.S. and Ph.D. degrees in Computer Science from Cornell University, Ithaca, NY, in 1985 and 1987, respectively. She has been on faculty at Department of Computer Science and Engineering, University of Minnesota, Twin Cities since 1987, where currently she is a professor. Her current research interests include numerical linear algebra, pattern recognition, information retrieval, data mining, and bioinformatics. She served on the editorial board of SIAM Journal on Scientific Computing, Society for Industrial and Applied Mathematics, from 1993 to 1999. Currently, she is on the editorial board of Mathematics of Computation, American Mathematical Society, BIT Numerical Mathematics, and Computational Statistics and Data Analysis, International Association of Statistical Computing, a special issue on numerical linear algebra and statistics. She has recently served on the committees of several meetings including the program committees for text mining workshop at SIAM conference on Data 
Mining for past several years. Since November, 2003, she has been at the National Science Foundation as a program director in the Devision of Computer \& Communication Foundations, the Directorate of Computer and Information Science and Engineering (CISE/CCF). 
Figure 1. Fingerprint images

Figure 2. The fingerprint and absolute magnitude images by FFT

Figure 3. Directional filter

Figure 4. $M_{\theta}$ of $p=2, a=1.2$ and $\theta=45^{\circ}$

Figure 5. The directional images

Table 1. The distribution of fingerprints in NIST database 4

Table 2. The prediction accuracies for the five-class classification problem (1)

Table 3. The confusion matrix

Table 4. The prediction accuracies for the five-class classification problem (2)

Table 5. The prediction accuracies for the four-class classification problem 

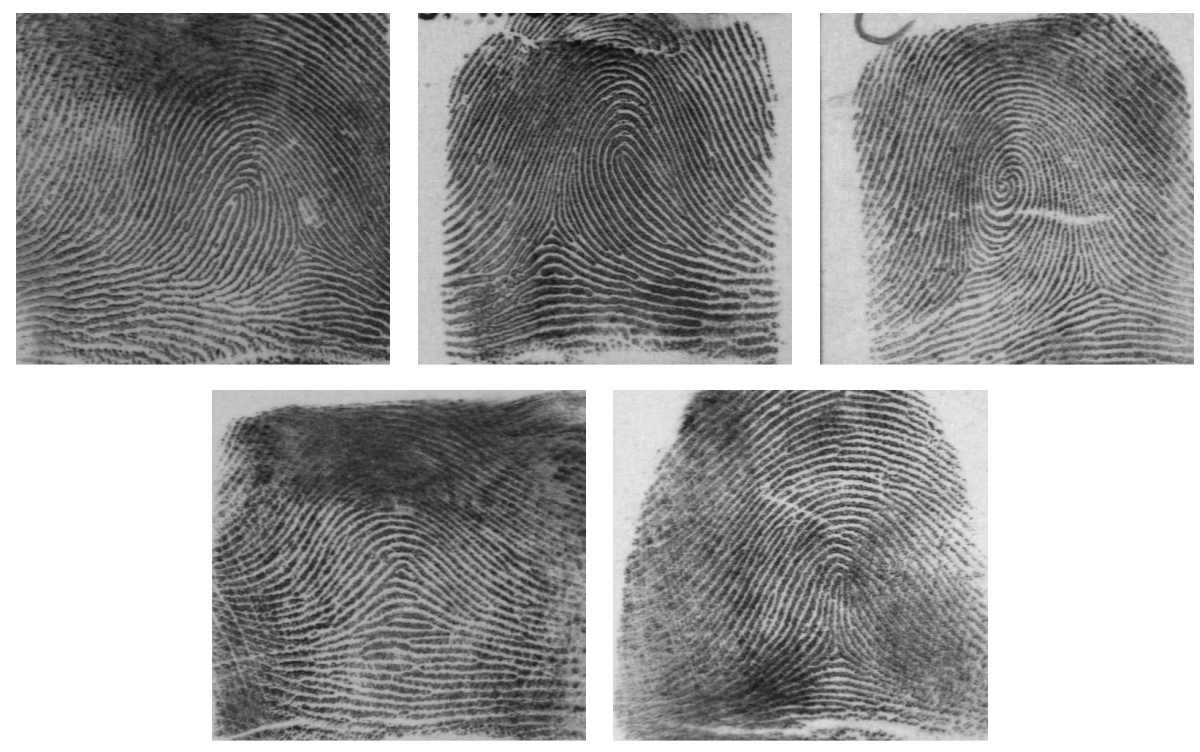

Figure 1: Fingerprint images. From the top left, class labels are left loop (L), right loop (R), whorl (W), $\operatorname{arch}(\mathrm{A})$, and tented $\operatorname{arch}(\mathrm{T})$.
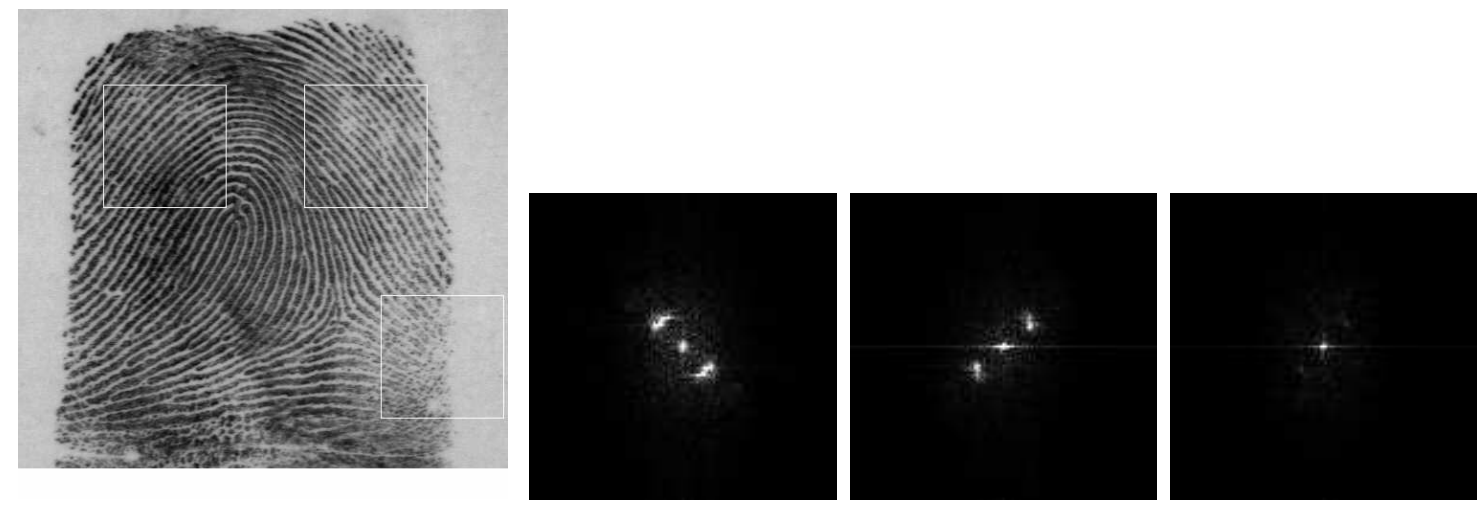

Figure 2: The fingerprint and absolute magnitude images by FFT. The values less than minimum value are displayed in black, the values greater than max/C are displayed in white, and values in between are displayed in intermediate shades of gray. The first and second pictures are FFT images of the top two squares in the fingerprint image, respectively $(\mathrm{C}=30)$. The last picture is the FFT image of below square in the fingerprint image $(\mathrm{C}=30)$. 


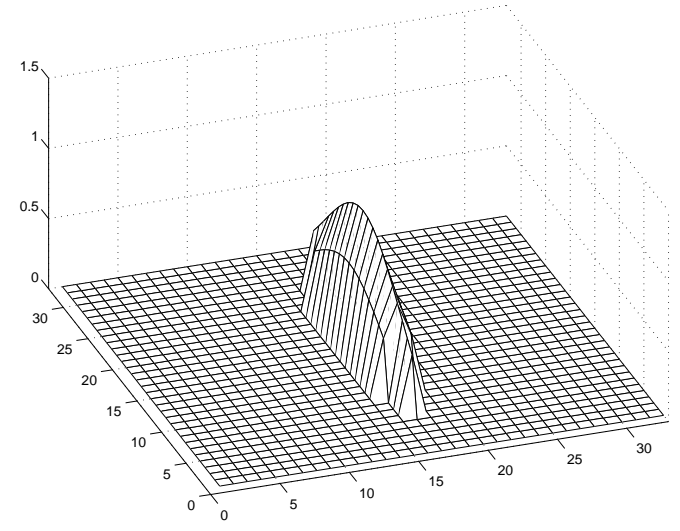

Figure 3: Directional filter $D_{\theta}$ of $p=16, a=1.5, b=32 / 3$ and $\theta=0$.

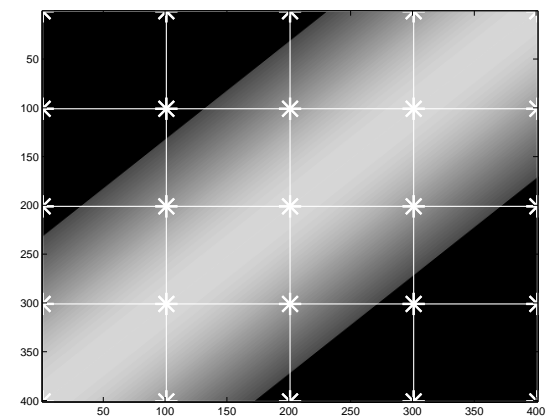

Figure 4: $M_{\theta}$ of $p=2, a=1.2$ and $\theta=45^{\circ}$ : The values are displayed in gray scale and pixel points of the size $5 \times 5$ are shown as $*$. 


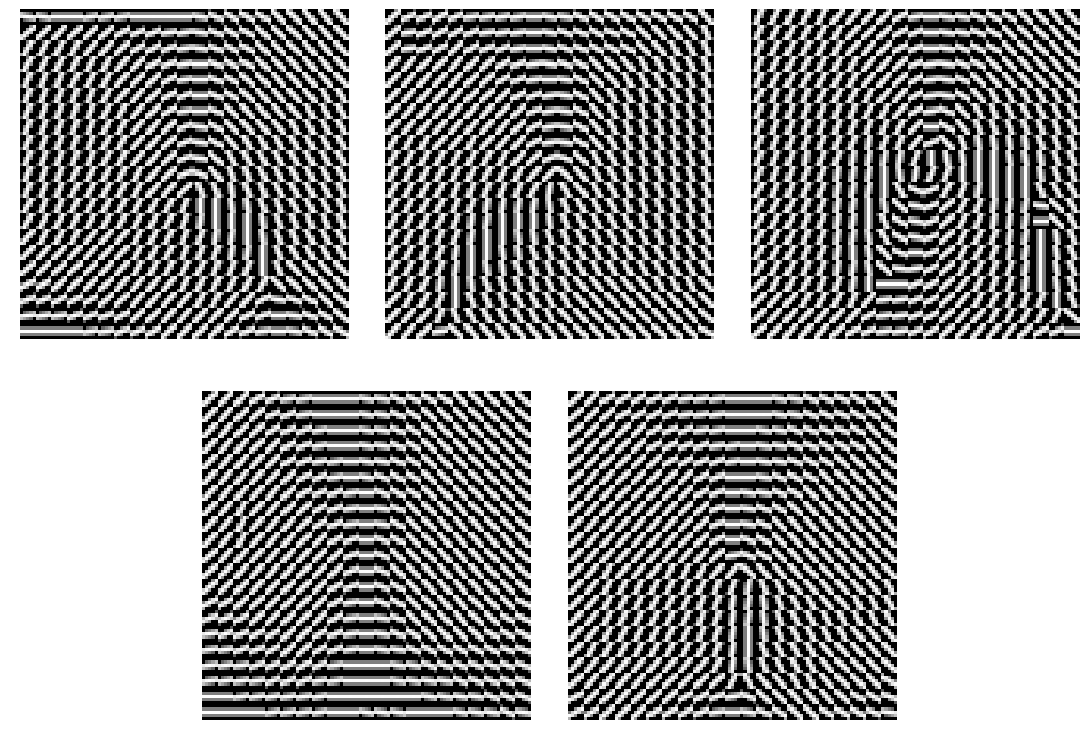

Figure 5: The directional images of size $105 \times 105$ corresponding to the fingerprints in Fig. 1. From the left, the class label is L, R, W, A and T.

\begin{tabular}{cccccc}
\hline & $\mathrm{L}$ & $\mathrm{R}$ & $\mathrm{W}$ & $\mathrm{A}$ & $\mathrm{T}$ \\
\hline \hline $\mathrm{L}$ & 756 & 0 & 2 & 0 & 42 \\
\hline $\mathrm{R}$ & 0 & 746 & 4 & 0 & 50 \\
\hline $\mathrm{W}$ & 2 & 6 & 792 & 0 & 0 \\
\hline $\mathrm{A}$ & 0 & 2 & 0 & 760 & 38 \\
\hline $\mathrm{T}$ & 166 & 238 & 0 & 150 & 246 \\
\hline
\end{tabular}

Table 1: The distribution of fingerprints in NIST database 4. Each row sums up to 800 according to the distribution based on the first class label. Within each row, the distribution of fingerprints which are cross-referenced to the second class is shown along the column.

\begin{tabular}{ccccc}
\hline rejection rate( $\%)$ & 0 & 1.8 & 8.5 & 20.0 \\
\hline \hline The proposed method & 90.7 & 91.3 & 92.8 & 95.3 \\
\hline From (Jain et al. 1999) & - & 90.0 & 91.2 & $93.5^{*}$ \\
\hline From (Yao et al, 2003) & - & 90.0 & 92.2 & 95.6 \\
\hline
\end{tabular}

Table 2: The prediction accuracies for the five-class classification problem (1). Rejection rate $19.5 \% *$ 


\begin{tabular}{c|ccccc}
\hline & \multicolumn{5}{|c}{ Assigned Class } \\
Actual Class & $\mathrm{L}$ & $\mathrm{R}$ & $\mathrm{W}$ & $\mathrm{A}$ & $\mathrm{T}$ \\
\hline \hline $\mathrm{L}$ & 361 & 3 & 2 & 8 & 14 \\
\hline $\mathrm{R}$ & 1 & 371 & 0 & 2 & 28 \\
\hline $\mathrm{W}$ & 9 & 20 & 366 & 2 & 2 \\
\hline $\mathrm{A}$ & 6 & 5 & 0 & 412 & 19 \\
\hline $\mathrm{T}$ & 12 & 8 & 1 & 44 & 304 \\
\hline
\end{tabular}

Table 3: The confusion matrix of the classification result by our proposed method for zero rejection rate.

\begin{tabular}{ccccc}
\hline rejection rate(\%) & 0 & 1.8 & 8.5 & 20.0 \\
\hline \hline The proposed method & 90.7 & 91.3 & 92.8 & 95.3 \\
\hline PCASYS + KDA/GSVD & 89.7 & 90.5 & 92.8 & 95.6 \\
\hline From (Jain et al. 1999) & - & 90.0 & 91.2 & $93.5^{*}$ \\
\hline From (Yao et al, 2003) & - & 90.0 & 92.2 & 95.6 \\
\hline
\end{tabular}

Table 4: The prediction accuracies for the five-class classification problem (2). Rejection rate $19.5 \% *$

\begin{tabular}{ccccc}
\hline rejection rate(\%) & 0 & 1.8 & 8.5 & 20.0 \\
\hline \hline The proposed method & 94.0 & 94.6 & 96.1 & 97.9 \\
\hline PCASYS + KDA/GSVD & 93.3 & 94.2 & 96.1 & 98.2 \\
\hline From (Jain et al. 1999) & - & 94.8 & 95.5 & $96.6^{*}$ \\
\hline From (Yao et al, 2003) & - & 94.7 & 96.6 & 98.4 \\
\hline
\end{tabular}

Table 5: The prediction accuracies for the four-class classification problem. Rejection rate $19.5 \% *$ 